\title{
Erratum to: Advancing drug delivery systems for the treatment of multiple sclerosis
}

\author{
Inna Tabansky ${ }^{1} \cdot$ Mark D. Messina $^{2,3} \cdot$ Catherine Bangeranye $^{3} \cdot$ Jeffrey Goldstein $^{2,3}$. \\ Karen M. Blitz-Shabbir ${ }^{2} \cdot$ Suly Machado $^{2,3} \cdot$ Venkatesh Jeganathan $^{4} \cdot$ \\ Paul Wright ${ }^{2}$ - Souhel Najjar ${ }^{2}$ - Yonghao Cao $^{4}$ - Warren Sands ${ }^{5,6}$. \\ Derin B. Keskin ${ }^{7} \cdot$ Joel N. H. Stern ${ }^{1,2,3,4}$
}

Published online: 19 February 2016

(c) Springer Science+Business Media New York 2016

\section{Erratum to: Immunol Res (2015) 63:58-69 DOI 10.1007/s12026-015-8719-0}

Unfortunately, the author names were incorrect in the Reference 86 in the original publication of the article. The corrected reference [86] is given below.

\section{Reference}

86. Price JD, Hotta-Iwamura C, Zhao Y, Beauchamp NM, Tarbell KV. DCIR2 + cDC2 DCs and Zbtb32 restore CD4 + T cell tolerance and inhibit diabetes. Diabetes. 2015;64:3521-31.

The online version of the original article can be found under doi:10. 1007/s12026-015-8719-0.

Joel N. H. Stern

jstern01@ rockefeller.edu; joel.n.stern@hofstra.edu

1 Department of Neurobiology and Behavior, The Rockefeller University, New York, NY, USA

2 Department of Neurology, Hofstra North Shore-LIJ School of Medicine, Hempstead, NY, USA

3 Department of Science Education, Hofstra North Shore-LIJ School of Medicine, Hempstead, NY, USA

4 Department of Autoimmunity, The Feinstein Institute for Medical Research, North Shore-LIJ Health System, Manhasset, NY, USA

5 School of Engineering and Applied Sciences, Harvard University, Cambridge, MA, USA

6 Department of Medicine, University of Pittsburgh Medical Center, Pittsburgh, PA, USA

7 Department of Cancer Immunology and AIDS, Dana FarberHarvard Cancer Institute, Boston, MA, USA 